because it is hospital policy. Do their employees wash their hands as often as the infection control department asks them to? We also expect our employees and medical staff to comply with hospital policy but take into account the likelihood that there will be less-than-perfect compliance. I do not consider asking an employee to wear a mask for an entire shift to be the same as asking the employee to don a mask intermittently when entering the room of a patient on respiratory precautions. Even in the operating room environment, it is rare for an individual to wear a mask continuously for an entire shift. Indeed, in the discussion section of the article, the authors report that one of their var- icella-exposed employees did not wear the mask as long as directed and developed varicella while unmasked, exposing other employees and patients. I contend that their own experience with "mistakes" is quite likely to be repeated.

Haiduven and her colleagues point out that only 4 of 45 , or about $10 \%$ of their employees who reported exposures, developed symptomatic varicella infection. This parallels our experience; we also have a conservative definition of employee exposure. Yet of the four patients at their hospital who did develop varicella, one managed to expose others because of poor compliance with the masking strategy. Although this may be an acceptable failure rate in some settings, for many hospitals and clinics, the costs associated with even occasional failures may exceed the benefits.

Haiduven et al present an interesting challenge to traditional management of varicella exposures among healthcare workers. I would caution that, in the search for cost savings or convenience, we must not embrace options that should work in theory without carefully considering how they might fail in practice.

Edward J. O'Rourke, MD Children's Hospital Boston, Massachusetts

\title{
Joint Commission A pproves Format of Quality Performance Reports for Healthcare Organizations
}

\section{by Gina Pugliese, RN, MS Medical News Editor}

Joint Commission on Accreditation of Health Care Organizations (JCAHO) recently approved the format and content for organizationspecific performance reports on healthcare quality. JCAI-IO, the largest healthcare accrediting body in the United States, began releasing these reports in mid-November as part of its expanded public disclosure policy. Reports will be available for approximately 1,500 hospitals, home care, mental healthcare, long-term care and ambulatory care organizations surveyed after January 1, 1994. A full catalog of performance reports on the nearly 11,000 organizations accredited by JCAHO will not be completed until the end of 1996, which will mark the end of the first 3-year accreditation cycle under the revised public disclosure policy.

Each performance report will include 1) a brief overview of the content of the report; 2) the accreditation decision and date; 3 ) the organization's overall evaluation score in comparison to like organizations surveyed; 4) the organization's numerical scores in a series of specific identified performance areas in comparison to like organizations surveyed; and 5) identified performance areas for which recommendations for improvement were made.

JCAHO's recent action was the result of more than a year of extensive field research to ascertain the interests and needs of consumers, practitioners, healthcare organizations, professional associations, and purchas- ers regarding performance information about surveyed organizations.

The American Hospital Association has actively supported the public release of hospital-specific quality data, provided that the information released is useful to the public and fair to hospitals. Healthcare facilities would be notified of the public request for the report. Those requesting the facility performance report would also receive a descriptive document explaining the information in the report and its appropriate use and limitations. Reports from facilities that are contesting their survey report will not be released until the review is complete. The cost of the report will be $\$ 30$. Reports will be available through the Customer Service Center (telephone [708] 9165800). 